\title{
Ein Interview mit Alfred Franz
}

\author{
Alfred Franz \\ Vormals \\ Österreichisches \\ Statistisches Zentralamt \\ (ab 2000 Statistik Austria)
}

Norbert Rainer

Vormals

Österreichisches

Statistisches Zentralamt

(ab 2000 Statistik Austria)

\section{Josef Richter}

\author{
Vormals \\ Bundeskammer der \\ gewerblichen Wirtschaft, \\ Wirtschaftskammer Österreich
}

\begin{abstract}
Das Interview mit Alfred Franz wurde von Norbert Rainer und Josef Richter am II. Februar 2020 in Wien durchgeführt. Es beleuchtet die Entwicklung der Volkswirtschaftlichen Gesamtrechnung in Österreich seit I965 und insbesondere den geänderten Stellenwert der Amtlichen Statistik nach dem Beitritt Österreichs zu EU. Ein weiteres Thema ist die Entwicklung der Österreichischen Statistischen Gesellschaft in den letzten Jahrzehnten.
\end{abstract}

Alfred Franz war von 1965 bis 2002 im Österreichischen Statistischen Zentralamt (später Statistik Austria), ab 1976 stellvertretender, von 1982 bis 1994 Leiter der Abteilung für Volkswirtschaftliche Gesamtrechnung, danach bis 2002 Leiter der Abteilung Sozialstatistik (einschließlich Internationale Vergleiche und Regionale Gesamtrechnung). I984 Ernennung zum Hofrat. Von I989 bis 2009 Honorarprofessur für Wirtschafts- und Sozialstatistik an der Universität Wien, Vorlesungen an der Universität Innsbruck und der Technischen Universität Wien. Mitarbeit an zahlreichen internationalen Projekten, insbesondere auf dem Gebiet der Berechnung von Kaufkraftparitäten und Realvergleichen (ECE, WB, OECD, EU), zu Tourismus-Konten (WTO, OECD, EU) und zur „Hidden Economy“ (OECD). Tagungsbeiträge und Monographien zu den angeführten Themenkreisen.

Geschäftsführender Sekretär der Österreichischen Statistischen Gesellschaft von ${ }_{9} 82$ bis I995\% bis 2008 Mitglied des Vorstandes. 200I Verleihung der Ehrenmitgliedschaft.

Hauptanliegen waren stets Systemfragen der Volkswirtschaftlichen Gesamtrechnung aller Art und die Entwicklung von Satellitenkonten (z.B. Gesundheit, Haushaltsarbeit, Tourismus), spezifische Methodenfragen und insbesondere die Behandlung des „Staates“ in der Volkswirtschaftlichen Gesamtrechnung.

Keywords: interview, national accounts, official statistics. 
Josef Richter: Einen schönen guten Nachmittag. Es freut mich sehr, dass wir heute die Reihe von Interviews, die wir für die Zeitschrift der Statistischen Gesellschaft begonnen haben, fortsetzen, und zwar mit einem Gespräch mit unserem Ehrenmitglied Dr. Alfred Franz, einem Pionier der Volkswirtschaftlichen Gesamtrechnung (VGR), aber nicht nur dieser Art von Statistik. Als Mit-Interviewer konnten wir Dr. Norbert Rainer gewinnen, einen langjährigen Weggefährten von Dr. Franz in der Amtlichen Statistik.

Wir fangen am besten mit dem Lebenslauf an: Das haben wir immer so gemacht, und da stellt sich mir die Frage: Wie gerät einer, der im Innviertel geboren ist, in die Amtliche Statistik, und dort ausgerechnet in die VGR?

Alfred Franz: Darf ich zunächst auch die Interviewer als Kollegen von seinerzeit - von mir aus natürlich besseren Zeiten - herzlich begrüßen und das Wiedersehen feiern. Also, wie kommt einer wie ich in die VGR-Gasse? Das ist eine nicht mit einem Satz zu beantwortende Frage. Ich hole nicht so weit aus, aber es gab mich einmal auf der Universität Wien, da habe ich nach einem Probier-Semester auf der Philosophischen Fakultät ein Jus-Studium begonnen. Mit Verzögerungen aus diversen Gründen, z.B., weil ich zeitweilig gearbeitet habe, oder weil ich zwischendurch einmal meine Frau geheiratet habe. Kam dann wieder zurück in die Jus-Gasse, war aber davon nicht mehr so angetan, habe aber im sogenannten Staatswissenschaftlichen Studienabschnitt die Nationalökonomie kennengelernt. Das war für mich wirklich eine Entdeckung, die mich fasziniert hat, und wo ich gewissermaßen fachlich "picken“ geblieben bin. Da gab es zum Schluss auch ein Rigorosum, abgenommen damals von Prof. Mahr, dem das so gefallen hat, dass er mich zu Dr. Lothar Bosse (dem späteren Präsidenten des Österreichischen Statistischen Zentralamts) in die „Statistik“ weiterempfohlen hat. Es folgte ein ausführlicheres Gespräch mit Dr. Bosse, und so kam ich gleich weiter zur VGR, jener prominenten Novität im Bereich der amtlichen Wirtschaftsstatistik. Was denen so an mir gefallen hat, dass sie mich für die VGR ausersehen hatten, das weiß ich nicht, aber es ging irgendwie schon um den nationalökonomischen Rahmen. Das war also der Hintergrund, der mit dem Innviertel nichts zu tun hat. Aber ich selber habe mit dem Innviertel nach wie vor sehr viel zu tun, das ist ein Geburts-Genom.

Josef Richter: Wann begann dann die Tätigkeit in der Amtlichen Statistik?

Alfred Franz: Das war alles i965. Das war eine Zeit, zu der VGR in Österreich eigentlich noch nicht wirklich etabliert war. Was damals gemacht worden ist, entstand vor allem am Österreichischen Institut für Wirtschaftsforschung (WIFO); beim Amt war nur der „Öffentliche Sektor“. Das wurde damals alles von sehr geschickten Leuten gemacht, die haben ihr Geschäft wirklich sehr gekonnt aufgezogen, schlank und zielbewusst. Um diese Zeit ist aber auch der Ruf nach einer Amtlichen Gesamtrechnung laut geworden. Das sollte also bei uns im Statischen Zentralamt passieren. Es waren aber keine Fachleute da, auch unsere Chefin, die Frau Hofrat Dr. Auguste Klamecker, eine hochgewachsene Person, echt eindrucksvoll in ihrer Erscheinung, die hat uns aber durch VGR Kenntnisse nicht recht zur Hand gehen 
können. Eher hat sie gewissermaßen mit uns gelernt. Wir haben alles, im Stile von „Learning by doing“ und „Doing by learning“ versucht hinzukriegen. Das hat natürlich nicht über Nacht funktioniert, sondern es gab Anlaufschwierigkeiten und Probleme genug, bis wir Schritt für Schritt gegen Ende der 6oer Jahre soweit waren, dass wir regulär publizieren konnten, bis dahin war es bestenfalls Stückwerk.

Josef Richter: Das war doch auch die Zeit, zu der Hofrat Dr. Anton Kausel vom WIFO in die Amtliche Statistik gexvechselt ist?

Alfred Franz: Zu dieser Zeit war er noch nicht bei uns, wir haben mit ihm aber zu tun gehabt, das war im WIFO das Team Dr. Nandor Nemeth und Dr. Kausel - sehr geschickte, statistisch versierte Fachleute. Da konnten wir wirklich viel lernen.

Alles in allem haben wir also Mühe gehabt, uns in die VGR hinein zu arbeiten, praktisch und auch theoretisch zugleich, das war vielleicht damals positiv und negativ in einem. Wenn wir eine kompetentere Führung gehabt hätten, wäre es schneller gegangen, dafür hätten wir aber vielleicht nicht so viel Theoretisches mitbekommen.

Josef Richter: Hat es nicht auch damals schon Meilensteine gegeben, wie zum Beispiel die InputOutput Tabelle 1964?

Alfred Franz: Ja, so was gab's tatsächlich. Wenn ich heute an diese Input-Output Tabelle denke, dann würde ich mich dafür nicht gerade genieren, das war für uns ein wichtiger Einstieg. Heute würde man das sicher viel anspruchsvoller und viel gekonnter machen, wenn ich etwa an die Frage denke, wie man mit Handelspannen und Import- und Exportströmen in der Statistik umgehen muss. Wir waren uns dessen gar nicht bewusst, was da eigentlich erfordert sein würde, und haben das mehr oder weniger forsch und fröhlich einfach „gemacht“. Ja, dann gab es eine Studienreise, am Anfang der zoer Jahre, in führende Input-Output Länder in Europa, da haben wir manches gelernt. Eine wirkliche Input-Output Statistik zu machen, das geht nicht über Nacht. Da braucht es gute Vorbereitung und gut eingearbeitete Leute. So gesehen, war die Tabelle ig64 eine Pionierarbeit.

Josef Richter: Wie waren denn damals die technischen Voraussetzungen? Ich erinnere mich z.B. mit Vergnügen an deinen virtuosen Umgang mit der elektrischen Handrechenmaschine.

Alfred Franz: Da war ich vielleicht deswegen so gut, weil ich das alles mit „Links“ gemacht habe. Man musste natürlich viel addieren damals. Da gab es noch kaum Computer, die gab es irgendwo im Keller, noch nicht auf den Schreibtischen.

Josef Richter: Dann bist du relativ bald stellvertretender Leiter der Abteilung Volkswirtschaftliche Gesamtrechnung geworden und später für viele Jahre deren Leiter.

Alfred Franz: Ja das kann man ungefähr mit den joer Jahren beginnen lassen. Und ich habe mir Mühe gegeben, dass wir eine gute und anständige Volkswirtschaftliche Gesamtrechnung aufbauen. Was wir in der Zeit meiner seinerzeitigen Leitung nicht geschafft haben, war eine kurzfristige Rechnung, eine Quartalsrechnung. Das ist also beim WIFO geblieben. Was wir auch von unserem Auftrag her schon gar nicht anzugehen hatten, war eine prospektive, prognostische VGR. Aber die Zusammenarbeit mit dem WIFO war immer problemlos und gut. Und das WIFO war vielleicht auch froh, dass sie einen fetten Happen des VGR-Berichtswesen bei sich 
behalten konnten. Das war sicher wichtig für die wirtschaftspolitische Analyse und Beratung und das ganze Standing des WIFOs.

Norbert Rainer: Wurde nicht damals auch schon mit dem Aufbau der regionalen Gesamtrechnung begonnen?

Alfred Franz: Die regionale Gesamtrechnung wurde nicht parallel mit der nationalen VGR aufgebaut. Ich bin jetzt überfragt, wenn ich dazu ein Jahr nennen soll, also wann die regionale Gesamtrechnung wirklich begonnen hat. Die ersten Schritte auf dem Gebiet habe ich mit meinen Leuten schon gemacht, das stimmt schon. Ich weiß nicht, wie man da heute vorgeht, aber mein Ansatz war damals sehr statistiknahe. Ich habe versucht, eine Art Modell über die Basisstatistiken drüber zu legen, wo man die regionale Gesamtrechnung herausfiltern konnte, ohne dass man sehr viele ExtraDaten gebraucht hätte. Unter dem Titel VGR hat man damals ja überhaupt keine Erhebungen machen können.

Josef Richter: Vielleicht noch ein zwei Worte zu den Datengrundlagen, die ja einerseits sehr spärlich waren. Andererseits, wenn ich mich so an 1970 und danach erinnere: Konnte damals die VGR nicht die wirtschaftsstatischen Erhebungen - was die Konzepte betrifft - wesentlich gestalten? Oder erinnere ich mich da nicht richtig? Die sogenannten Jahreserhebungen und die sogenannten Bereichszählungen waren doch von den Datenbedürfnissen der Volkswirtschaftlichen Gesamtrechnung dominiert!

Alfred Franz: Ja, ja, das stimmt schon. Man kommt da sehr eng an die legistischen Grundlagen der Statistik von damals heran. Mit dem Bundesstatistikgesetz 1965 ist sicher vieles besser geworden als in der Vergangenheit. Das hat dann aber erst im Laufe der Jahre gewirkt, und schließlich dazu geführt, dass uns das statistische System mehr oder weniger flächendeckend mit Daten bedient hat. Das war ja nicht sofort so, wenn es mit I965 ein Bundesstatistikgesetz gegeben hat, dass dann 1966 schon alle Daten verfügbar gewesen wären. Das hat sich erst eingespielt im Laufe der zoer Jahre, als sukzessive dann zum Beispiel die Großgewerbe-Statistik und die statistischen Erhebungen auf der Basis von Stichproben im Gewerbe und über größere Zeiträume gehende Erhebungen, wie die Konsumerhebung usw. nachgeschickt worden sind. Das war nicht von heute auf morgen zu haben, obwohl das Bundesstatistikgesetz 1965 insofern bemerkenswert ist, weil es das erste Gesetz war, in dem der Begriff "Volkswirtschaftliche Gesamtrechnung" überhaupt vorgekommen ist.

Norbert Rainer: Die Entwicklungen der Volkswirtschaftlichen Gesamtrechnung in Österreich hast du ja ganz kräftig mitgesteuert und geprägt. Zusätzlich warst du immer schon sehr stark auf dem internationalen Gebiet tätig. In Gremien, bei Sitzungen, bei Arbeitsgruppen von OECD und der UN, du hast viele Studien zu Themen der VGR, aber auch zu Satellitensystemen in anderen Bereichen vorgelegt, wenn ich etwa an die Tourismuskonten denke.

Alfred Franz: Das ist ein guter Punkt, aber wieso kommt es dazu, dass die Österreicher auf einmal gesuchte Leute waren? Da muss ich wieder zurück gehen an den Ausgangspunkt von vorhin. Unser Beginnen in der VGR war von Anfang an stark ausgerichtet an den theoretischen Konzepten der VGR, wir haben unser Handwerk mit den Büchern in der Hand gelernt. Diese Mentalität, diese Einstellung, die hat mich persönlich nie verlassen. Ich habe immer auch hinter die Kulissen schauen wollen, was steckt denn da dahinter? Was ist denn das Gesamtkonzept, wie kommt 
es überhaupt zustande, dass man da zu konsistenten Daten kommt? Das war also immer ein Punkt, gleich von Anfang an, und er ist auch irgendwie widergespiegelt in meinen Publikationen zu dem Thema VGR. Das war vielleicht auch der Grund, dass man international aufmerksam geworden ist, da ist Einer, der könnte zu dem und jenem etwas sagen. Da ist man dann eingeladen worden, teils zu den Sitzungen, teils zu Beratungstätigkeiten in verschiedenen Ländern und zu Studien daheim. Es hängt damit zusammen, dass wir in Österreich diesen Stil hatten, den es so - glaube ich heute nicht mehr gibt. Bis herauf zum Jahr 2000 waren wir immer auch stark theoretisch orientiert.

Josef Richter: Und die vielfältigen Publikationen auf dem Gebiet haben dann auch zu akademischer Lehrtätigkeit geführt.

Alfred Franz: Ja, schon. Ich habe Lehraufträge schon früh an den Universitäten in Wien, auf der TU Wien gehabt, in weiterer Folge auch an der Universität Innsbruck. Wenn man immer brav Lehrtätigkeit ausübt und auch publiziert, dann ist es fast unvermeidlich, dass irgendeine akademische Graduierung nachfolgt. Ich hatte es aber nicht auf eine akademische Karriere angelegt, die Ernennung zum Honorarprofessor kam eher überraschend, aber sie hat mich umso mehr gefreut, weil es doch auch eine Anerkennung war für eine ganze Menge von wissenschaftlichen Publikationen. Ich habe anlässlich der Vorankündigung des heutigen Termins einmal geschaut, wie ist das mit der Bibliothek zuhause? Da komme ich ca. auf 30 Meter Länge. Davon ist immerhin ein Meter authentische Lektüre aus meiner Feder. Wenn ich da heute so hineinschaue, dann denke ich mir, das gibt es gar nicht, dass ich das alles geschrieben habe. Das ist oft wie ein fremdes Land, ein anderer Kontinent. Zum Beispiel diese Broschüre „Volkswirtschaftliche Gesamtrechnungen. Das statistische System der Makroökonomie“, da würde ich heute sagen, das war wirklich ein Opus Magnum. Wenn man mal 8o vorbei ist, dann schaut man auf die eigene Vergangenheit, ich möchte fast sagen, mit einer großen Gelassenheit und regt sich gar nicht mehr auf, wenn dieses oder jenes nicht mehr in Erinnerung so voll da ist.

Josef Richter: Warst du damals eigentlich der Erste aus der Amtlichen Statistik, der auf Universitäten tätig war?

Alfred Franz: Nein, das glaube ich nicht. Ich glaube Dr. Bosse hat immer schon eine Vorlesung gehabt, seinerzeit, als er Vizepräsident des Statistischen Zentralamts war.

Josef Richter: Generell war das schon ein sehr wichtiger Schritt, um diese Kluft zwischen der akademischen Welt und der Amtlichen Statistik ein bisschen zu schließen.

Alfred Franz: Vielleicht ist das der wichtigste Punkt, dass VGR als Thema damals auf den Universitäten noch präsent war. Das ist ja heute so nicht mehr der Fall. Damals gab es noch Vorlesungen mit dem Titel „Volkwirtschaftliche Gesamtrechnung“, oder „Input-Output Statistik“. Damals gab es auf den Universitäten diese Diskussion der grundlegenden Konventionen hinter den Regeln der VGR und ihren zentralen Begriffen, wie z.B. jenem der „Produktion“, und es gab Studenten, die sich damit auseinandergesetzt haben. Es gab dann und wann sogar Leute, die in dem Bereich ihr Magisterium gemacht haben. Ob das heute überhaupt noch der Fall ist? Ich bin mit den Unis nicht mehr auf Tuchfühlung, kann das also nicht beurteilen.

Josef Richter: Für mich war immer sehr charakteristisch für den Wissenschaftler Alfred Franz, dass er sich nicht mit isoliert in der Welt stehenden statistischen Einzelaussagen begnügt hat, 
sondern immer einen starken Hang zu Gesamtrechnungen, zu großen Konzepten gehabt hat, und zwar auf verschiedenen Gebieten. Ich denke zum Beispiel, etwas was nicht unbedingt in den engsten Bereich der VGR gehört, an die wirklich epochalen Konzepte zu einer integrierten Lohn- und Einkommenssteuerstatistik. Eine Statistik, die für damalige Verhältnisse, etwas ebenso Naheliegendes wie Sensationelles war.

Alfred Franz: Mag schon sein, dass so etwas wirklich aus der Gedankenwelt der Gesamtrechnung leichter hervorgegangen ist. Diese Art Gedanken, dass man integrieren muss, dass man Dinge zusammenführen muss, gegeneinander abwägen und vielleicht auf einen Nenner bringen muss, womöglich über die gleiche statistische Einheit, und dann bekommt man interessantes Neues, was vorher so gar nicht existiert hat. Das wird schon mit der Denkweise der Volkswirtschaftlichen Gesamtrechnung zu tun haben.

Manchmal war es auch eine aus der Not geborene Notwendigkeit, keine Tugend. Etwa die vielen internationalen Vergleiche, wie die Berechnung von Kaufkraftparitäten. Diese Berechnungen haben zu einer Zeit begonnen, da gab's im planwirtschaftlich organisierten Osten kaum je Kontakte mit dem Westen. Aber es gab immer schon ein bisschen was von der Sorte in Polen und Ungarn, die waren nicht so linientreu, haben sich getraut und die haben's gemacht. Die Preis-Vergleiche haben aber im Laufe der Jahre dann so expandiert, dass sie als Berechnung von Kaufkraftparitäten zum regulären Programm der UNO geworden sind. Inzwischen gehört es auch zum regulären Programm der Europäischen Union, dass man einfach alles und jedes mit kaufkraftbereinigten Preisen vergleicht. Ich würde heute sagen, es ist fast ein bisschen übertrieben, was da heute alles verglichen wird, aber damals war es eine Pionierleistung. Man wusste ja wenig darüber, wie die ökonomischen Verhältnisse zwischen Ost und West wirklich waren. Vorher waren das nur Urteile von Autofahrern, die ein Gefühl gekriegt haben, wieviel kostet denn die Flasche Vyborowa in Polen im Vergleich zu Österreich. Wir haben systematisch verglichen, zunächst nur den privaten Konsum, in weiterer Folge auch die Staatsaktivitäten. Ja, das ist ein großes Ding geworden. Ich habe es nie so besonders geliebt, aber wir Österreicher waren da gefragt.

Josef Richter: Zu diesen großen Systemen, gehört doch auch diese Entwicklung einer Öko-VGR für Österreich mit dem Input-Output Kern. Ein Konzept, das, glaube ich, wirklich bahnbrechend war und das leider nicht den ganz großen Widerhall international gefunden hat, den es verdient hätte. Es gibt dazu eine schöne Publikation von Alfred Franz: „Entwicklung einer Öko-VGR in Österreich. Input- Output als Alpha und Omega “.

Alfred Franz: Ja, eine wirkliche Öko-VGR, so etwas haben wir in meiner Zeit nicht geschafft. Erstellt wurde ein Rahmen: Was könnte man da tun? In welchen Kontexten? In welchen begrifflichen Konzeptrahmen muss man das stellen? Ich habe immer wahnsinnig gern sowas aufgegriffen, schon früh, sobald sich abgezeichnet hat, dass es notwendig werden wird, aber ich konnte leider nicht alles selber tun: Konzepte machen und die Daten dazu liefern.

Josef Richter: Die große Stärke war doch, diese Konzepte zu erstellen. Ich denke beispielsweise an den volkswirtschaftlichen Wert der Hausarbeit, eine weitere große konzeptionelle Arbeit.

Alfred Franz: Da habe ich auch mehr persönlichen Anteil genommen, wo ich ja auch verheiratet bin und daher recht gut weiß, was Hausarbeit auch dann bedeutet, wenn 
man sie nicht selber macht. Ja, das hat mich als Thema mehr fasziniert als die ÖkoVGR, die damals eigentlich noch ein Waisenkind war, um das sich fast niemand angenommen hat. Die informelle Wirtschaft, die war damals plötzlich ein TopThema.

Josef Richter: Eines der großen Themen, mit denen du dich immer auseinandergesetzt hast, war die Frage nach der Rolle der Amtlichen Statistik.

Alfred Franz: Das ist die Frage nach „was tut und macht die Amtliche Statistik?" Was sie insgesamt tut, das geht mich nicht so viel an. Aber es geht den Gesamtrechner natürlich was an, ob seine Bedürfnisse im vollen Umfang bedient werden. In der Pionierzeit ist das sukzessive immer mehr und mehr so geworden, und immer besser. Diese Tendenz, mehr und mehr Daten bereit zu stellen, zu erzeugen, anzuordnen, zu liefern, die ist nicht nur geblieben, sondern dann nahtlos übergegangen in die statistische Welt der Mitgliedschaft bei der Europäischen Union. Und wenn ich mir das heute überlege, komme ich doch ein bisschen ins „Philosophieren“. Es gibt meines Wissens keine Diskussion, ob die Grenzen der Statistik schon erreicht sind oder man sie gar schon überschritten hat. Wie sieht jene Welt aus, die jenseits dieser Grenzen liegt? Wo müsste man sagen, das geht die Statistik eigentlich nichts mehr an, denn das ist zu subjektiv oder es ist zu singulär?

Josef Richter: Was ist das „Wesen “, das ist immer die Frage. Ich erinnere mich an eine Publikation mit dem schönen Titel „Von den ontologischen Konzepten in der amtlichen Statistik“.

Alfred Franz: Naja, das ist es. Aber wenn es Ontologie ist, dann ist es jetzt wirklich schon Philosophie. Ich habe schon seinerzeit eine reserviertere Position vertreten, und heute würde ich es umso mehr tun. Es gibt leider keine Diskussion, wo die Grenzen der Statistik verlaufen sollen. Wir haben heute die EU, die hat einen organisatorischen Standpunkt, einen technokratischen Standpunkt, einen bürokratischen Standpunkt, von dem aus man keine Grenzen der Statistik sieht, die wollen das immer weitermachen. Und wenn es noch kein Thema für Statistik ist, dann, oha, dann muss man mal Statistik machen und dann können wir weitersehen. Mir ist keine ontologische Diskussion dieser Art untergekommen, dass man sich von der EU-Seite in dieser Richtung selbst disziplinieren möchte.

Josef Richter: Dazu passt ja wunderbar, also abgesehen von den Landwirtschaftsförderungen, dass in der EU die umfangreichste Förderung die Regionalförderung ist. Und die basiert fast ausschließlich auf Ergebnissen bzw. abgeleiteten Kriterien der regionalen Volkswirtschaftlichen Gesamtrechnung. Wenn man sich die regionale Gesamtrechnung und ihre Aussagekraft vor Augen führt, ist das ein kühnes Unterfangen, um es mal so zu sagen. Und da fällt mir ein so wunderschöner Aufsatz von Alfred Franz ein, mit dem Titel „Regionale Gesamtrechnungen: Dürfte man, was man tut? Tut man was man dürfte?", lange bevor die EU alle diese Regionalförderungen auf Basis der regionalen Gesamtrechnung umgesetzt hat. Ich fürchte nur, diese Reflexionen haben nur wenig bewirkt.

Alfred Franz: Ja, das ist ein gutes Beispiel für die Problematik, die natürlich dort besonders prekär wird, weil es um so viel Geld geht. Wir haben ein Nachbarschaftsbeispiel, das ist Bratislava, eine der „reichsten“ Städte der EU, weil sich dort ein paar Unternehmen angesiedelt haben, die groß produzieren. Aber wenn das zum Schlüssel für die Verteilung von Regionalmitteln wird, dann ist das mit Händen zu greifen, dass das nicht richtig ist, dass man doch was anderes meinen würde, wenn es um regionale Fragen geht. Aber da gibt es nicht nur von mir, sondern auch von 
Josef Richter interessante Überlegungen, was im strengeren Sinne „statistisch noch machbar" ist. Man muss also sehr vorsichtig sein, ob man mit solchen „Daten“ nicht Missverständnisse generiert statt eines besseren Verständnisses und eines Beitrags zu einer sachgemäßen Lösung.

Josef Richter: Da ist tatsächlich ein ganz großes Problem der EU-Statistik angesprochen. Ich glaube, man müsste zunächst einmal ganz klar unterscheiden, zwischen dem, was man wissen kann, und dem, was auf einer schlichten Konvention beruht.

Alfred Franz: Ja, das ist es!

Josef Richter: Mit Konventionen könnte man ja leben. Nur wenn man die Einleitungen zu EUVerordnungen liest, wird da immer unterstellt, man könnte zu gewissen Fragestellungen etwas gesichert wissen. Kannst du das so bestätigen?

Alfred Franz: Kann ich nur unterschreiben. Leider gibt es aber gerade zu diesem Punkt, auch wenn deine systematische Kritik noch so berechtigt ist, eben noch immer keine Sachdiskussion. Anscheinend will eben niemand aufgreifen, was nicht opportun ist. Das heißt aber nicht, dass das Thema damit erledigt wäre, im Gegenteil. Um je mehr Geld es geht, umso prekärer wird es.

Josef Richter: Hat die EU zu einem echten Paradigmenwechsel geführt, oder wäre dies ohnehin passiert? Nämlich, dass statistische Ergebnisse unmittelbar politische und finanzielle Konsequenzen haben und die Grenzen des „statistisch Machbaren“ negiert werden.

Alfred Franz: Ich verfolge die Fachlektüre von heute nicht mehr lückenlos, nur, was so auf mich zukommt. Aber ich glaube nicht, dass es heute eine Diskussion auf der europäischen Ebene über die natürlichen Grenzen des statistischen Berichtswesens gibt. Ganz egal, auf welcher Ebene man das meint, es wird einfach nicht diskutiert, ganz im Gegenteil: Man bejubelt sich selber, weil man Daten für alles Mögliche hat. Dann kommen noch berühmte Inder und andere Nobelpreisträger und sagen, da muss man auch noch dies und das in ein Gesamtrechnungssystem einbauen, und niemand widerspricht wirklich, obwohl man zwar Manches zusammenzählen kann, aber man kann es nicht zusammengießen in ein systematisches Verständnis auf höherer Ebene, wo diese Dinge zusammenfinden. Da fehlt aus einer gewissen Opportunität heraus einfach die ehrliche Auseinandersetzung mit dieser Problematik. Da rührt sich kein Mathematiker, kein angewandter Statistiker, kein Gesamtrechner, kein EU-Experte.

Die VGR hätte eben nicht über alles zu berichten, sondern es ist ein ganz bestimmter Bericht, der hier allein sinnvoll ist. Aber wenn man dann anfängt, dass man das gleiche Konzept für sozialstatistische Zwecke verwendet, und weiß Gott was alles sonst noch, das überfordert sie einfach.

Josef Richter: Das ist ein gutes Stichwort. Nach deiner Tätigkeit in der VGR warst du ja auch in der Sozialstatistik tätig, bemerkenswert für einen geborenen Wirtschaftsstatistiker. Du hast sozusagen das Metier gewechselt.

Alfred Franz: Das war eine Entwicklung meiner letzten beruflichen Phase, damals noch im Statistischen Zentralamt, aber schon im Übergang zum heutigen System. Da haben verschiedene Dinge auch organisatorischer Art zusammengespielt, das würde den Bericht sprengen, wenn ich die hier auseinanderlege. Aus der VGR bin ich damals 
ausgezogen, weil sie für mich als theoretisches System mehr oder weniger doch erschöpft war. Insbesondere wurde aber durch den EU-Beitritt alles total verrechtlicht: Was sollte ich damit noch? Da wird es doch noch andere Herausforderungen geben.

Norbert Rainer: Eben neue Herausforderungen?

Alfred Franz: Größere Themen dieser Art gab es in der sozialstatistischen Umgebung sicher, sei es mit der neuen Gender-Thematik, der neuen Preisstatistik, von der EU auf uns zukommenden Anforderungen für internationale Vergleiche, die ich zwar nicht so geliebt habe, aber wo ich gesehen habe, da ist noch Konzeptuelles zu tun. Dafür also hatte ich die Seiten gewechselt.

Josef Richter: Der Hang zu großen Gesamt-Systemen, der wurde in der Sozialstatistik bislang ja nicht umgesetzt, obwohl schon Richard Stone, einer der Väter der VGR, ähnlich große geschlossene Systeme ja auch für die Sozialstatistik vorgeschlagen hat. Nur um z.B. die Publikation "Towards a System of Social and Demographic Statistics" der UNO zu nennen.

Alfred Franz: Ja, aber da war Sir Richard Stone schon lange tot. Wenn ich zur Frage, was habe ich da aufgegeben und was nicht, nochmal kurz konkretisieren darf: Eines habe ich nie aufgegeben, das ist die statistische Auseinandersetzung mit dem Thema Staat oder, allgemeiner gesprochen, mit der Nichtmarktwirtschaft. Das habe ich auch fortgesetzt in den Jahren, wo ich fast gar nichts mehr publiziert habe. Wie ein Versessener habe ich mich z.B. an einem Punkt verbissen, den könnte ich heute mit einem Wort erklären: Es gibt im Bereich des Staates viele statistische Einheiten, die nach den Regeln der EU marktmäßige „Produzenten“ sind, was aber bei der Berechnung des Beitrages des Staates zum Bruttoinlandsprodukt so nicht berücksichtigt wird. Auch so ein Punkt, von dem ich sagen würde, die Diagnose ist absolut richtig, er ist aber bei der EU so nie als Problem erkannt worden, nicht einmal bei der UNO. Man hat das nicht realisiert, dass unsere Berechnung nicht konsistent ist: Da ist so etwas wie ein kleiner Riss im Gebäude - hoffentlich bricht das nicht einmal zusammen! Nein, das glaub ich nicht, denn das darf ja nicht sein. Da kann man immer mal wo schrauben, da und dort was einziehen usw., das wird nicht zusammenbrechen. Aber ein Fehler liegt vor.

Norbert Rainer: Wir haben nun schon einige Male die EU-Statistik angesprochen. Die hat natürlich nach dem Beitritt Österreichs zur EU sehr viel Unruhe mitgebracht. Viele Anpassungen waren notwendig, und sor allem auch Erweiterungen der nationalen Statistik gegenüber dem, was wir vor dem Beitritt hatten. Du hattest auch die Aufgabe, die Umsetzung. der EU-Konzepte für Österreich zu betreuen und zu verhandeln.

Alfred Franz: Das war schon mit der Kandidatur zum Beitritt so, dass wir da in den Arbeitsgruppen der EU teilzunehmen hatten. Wir waren in Wirklichkeit aber immer ein bisschen hinten dran. Wir konnten nicht so gut liefern, wie die anderen Länder, die schon länger Mitglieder waren. Wir haben versucht aufzuholen: Daher war das dort vielleicht auch gar nicht das richtige Pflaster, unsere Probleme konzeptiver oder theoretischer Art unterzubringen. Wir hatten eher zu trachten, dass wir einfach bei der rechtzeitigen Bereitstellung der Zahlen, den Lieferverpflichtungen, Schritt gehalten oder aufgeholt haben.

Josef Richter: Du hast ja damals den statistischen Acquis, die Übernahme des EU-Rechts im Bereich der Statistik, verhandelt. 
Alfred Franz: Ja, das war mein Geschäft.

Josef Richter: Und konnte da mehr erreicht werden als ein paar Bezeichnungen zu retten, wie z.B. in den Klassifikationen? Dass es in der NACE, der EU-Klassifikation wirtschaftlicher Aktivitäten, nicht nur den Metzger gibt, sondern auch den Fleischhauer?

Alfred Franz: Es war sicher nicht die Situation, dass man dort große theoretische Probleme aufgreifen konnte. Man stieß auf ihre vorgegebenen Lösungen, alles schon wunderbar ausgeklügelt. Wenn wir dann dort verhandelt haben, so ging es um punktuelle Probleme, oder um das Bedürfnis nach einer bestimmten Klarstellung. Von einer grundlegenden, sozusagen akademisch befriedigenden Diskussion konnte nie die Rede sein.

Josef Richter: Da ging es also mehr um Übergangslösungen und solche Dinge.

Alfred Franz: Wir sind auch nie gefragt worden, ob wir einverstanden sind, oder was wir denn da für Grundsatzprobleme haben, das war nicht so. Es ging also eher vordergründig um das, was man jährlich und regelmäßig zu berichten hatte. Man durfte das kommentieren, man konnte vielleicht auch etwas einwenden, oder beantragen, dass ein uns wichtiger Punkt in eine Tagesordnung aufgenommen wurde. Es war aber nie eine Diskussion im grundsätzlichen Stil, wie es früher bei der OECD z.B. der Fall war. Dort wurden immer wieder Fragen von größerer Tragweite aufgegriffen, wie zum Beispiel: Was ist Produktion? Wie ist das mit der Haushaltsproduktion? Solche Fragen sind bei der OECD durchdiskutiert worden, aber bei EUROSTAT lagen sie schon wie fertig erledigte Dinge vor. Die Lösungen standen schon in den Manuals.

Norbert Rainer: Also, was schon in den Statistik-Verordnungen stand, war damit rechtswirksam. Durch den Beitritt mussten wir uns einfach an die damals schon gegebenen inhaltichen Vorgaben der EU-Statistik anpassen.

Alfred Franz: Ja, eben. Zu OECD Zeiten gab es solche Verordnungen nicht, keine wirklichen Standards in dem Sinn, die per se zu vollziehen waren, wie Rechtsnormen. In dem Moment, wo wir das ESVG 1995, das EU-System Volkswirtschaftlicher Gesamtrechnungen, zu übernehmen hatten, das schon existiert hat, als wir beigetreten sind, ist diese Diskussion einfach nicht mehr „top“ gewesen.

Josef Richter: Aber du hast immerhin noch auf wissenschaftlicher Ebene darüber meditiert. Da gab es den schönen Artikel „A Class-Society of National Accounts Compilers?”, genau über diese Problematik.

Nun aber zu ganz anderem Thema: Fast gleichzeitig mit der Übernahme der Leitung der Abteilung Volkswirtschaftliche Gesamtrechnung im damaligen Statistischen Zentralamt begann doch auch deine prägende Funktion in der Österreichischen Statistischen Gesellschaft. Du warst ja nicht weniger als 13 Jahre geschäftsführender Sekretär der ÖSG, so lange wie niemand vorher noch nachher. Und anschließend viele Jahre Vorstandsmitglied.

Alfred Franz: Ja, das ist eine Tatsache, die mich heute selbst erstaunt, dass da einer solange durchgehalten hat. Um was es damals aber eine Zeit lang auch gegangen ist, das war der Punkt: Wie kann man diese Gesellschaft am Leben erhalten? Die war zeitweilig nicht so gern gesehen bei Akteuren, auf die es angekommen ist, was z.B. das Quartier anbelangt, oder überhaupt um die größtmögliche Unterstützung im Amt. Da hatte 
ich schon eine spezielle Aufgabe und Verantwortung. Vor diesem Hintergrund ist damals auch die Idee der Arbeitskreise entstanden, die sich sehr vital entwickelt haben, das war sicher ein guter Punkt. Man musste halt schauen, dass es immer wieder ein Lebenszeichen gab, mal ein Vortrag, mal eine Veranstaltung in einem Arbeitskreis. Aber es war nicht leicht, in dieser Zeit für die Gesellschaft das Echo zu finden, das eine wissenschaftliche Gesellschaft braucht. Wir hatten zwar keinen wirklichen Mitgliederverlust, aber man muss auch sehen, dass viele Personen mit dem Eintritt in das damalige Amt quasi automatisch Mitglieder wurden. Diese Phase nicht so guter Stimmung war nach außen nicht unbedingt so zu bemerken. Und das Ganze hat dann allmählich doch wieder Fahrt aufgenommen, sicherlich auch durch die Verdienste der nachrückenden Vorsitzenden und durch die neuen Strukturen im Statistischen Amt. Heute sieht es insgesamt viel besser aus. Wenn ich heute in eine statistische Veranstaltung gehe, dann freue ich mich, wieviel junge Leute da auftauchen, die offenbar freiwillig zu dieser Gesellschaft gekommen sind und hier wirklich das Gefühl haben können, dass sie etwas zu bestellen haben und dass es interessante Angebote gibt.

Josef Richter: Aber es gab schon auch damals bemerkenswerte Aktivitäten, wenn ich z.B. an die internationalen Tagungen denke, die es damals gegeben hat. 1985 das Input-Output Symposium in Baden, 1986 die Tagung Statistik und Ausbildung in Linz, das 2. Input-Output Symposium in Baden im Jahr 1988, das Symposium Regionalstatistik in Graz, das waren durchaus Aktivitäten, die sich mehr als nur sehen lassen konnten. 1994 fand zudem gemeinsam mit der Deutschen Statistischen Gesellschaft die Statistische Woche in Wien statt.

Alfred Franz: Ja, und diese Aktivitäten haben Väter gehabt, von denen mindestens zwei hier sitzen. Es kommt ja wie immer sehr darauf an, wer tut sich das an? Sowas passiert ja nicht von allein, da muss schon immer wer dahinterstehen. Nun, einiges hat also die ganze Zeit ganz gut funktioniert, damit hatten wir sicherlich Echo, und das waren vielleicht auch die Eckpunkte, womit wir die schwierigeren Zeiten überbrückt haben.

Josef Richter: Es wurde ja doch auch unsere Zeitschrift revitalisiert, was das Verdienst von Norbert Rainer war. Es hat ja eine Zeit lang sogar eine eigene Schriftenreihe gegeben, etwas, das es weder vorher noch nachher wieder gab.

Alfred Franz: Ja, mit dem Letzteren ist es auf dieser Ebene leider nicht weiter gegangen, da erlebten wir auch mit dem Verlag, bei dem die Schriftenreihe, ferner Tagungsbeiträge und Monographien zu den angeführten Themenkreisen erschienen sind, so einige Probleme.

Norbert Rainer: Man muss aber auch sehen, dass wir die Erwartungen des Verlags, dass wir zur Informatik publizieren werden, was damals ja als Marktlücke gesehen wurde, nicht erfüllen konnten.

Josef Richter: Ein besonderes Anliegen war schon auch damals das Zusammenführen der drei Säulen, auf denen die Gesellschaft beruht: Wissenschaftliche, Amtliche Statistik und Angewandte Statistik, wie es in den neuen Satzungen von 2005 festgeschrieben ist.

Alfred Franz: Ich glaube, so etwas ist natürlich keine Entscheidung von heute auf morgen, das hat sich entwickelt, es ist eine gute Lösung, und lag so sicherlich schon in der Luft. Vor allem aber kamen wieder viel mehr akademische Leute zu uns, die sich wieder stärker engagiert haben, und so ist diese Gesellschaft heute wirklich wieder flott. 
In diesem Zusammenhang hätte ich gerne noch ein kleines „Anekdötchen“ erwähnt. Noch vor meiner (hochgeschätzten) Ehrenmitgliedschaft, aber wohl schon nach meiner Geschäftsführung hat mir die Gesellschaft eine Ehrennadel verliehen, ein Vorgang, der in den Annalen nie irgendwo Erwähnung gefunden hat: Die Nadel ist aus Gold, es steht drauf „ÖSG“, ich habe sie jetzt nur leider nicht bei mir. Kann vielleicht einer von euch dazu etwas sagen?

Norbert Rainer: Ja, auch ich habe die Nadel bekommen, für meine langjährige Tätigkeit als Schriftleiter. Die Nadel wurde insbesondere für Verdienste um die Gesellschaft vergeben, im Unterschied zur Ehrenmitgliedschaft, die primär eine Würdigung der wissenschaftlichen Verdienste um die Statistik darstellt. Trägst du die Nadel?

Alfred Franz: Nur am Opernball.

Josef Richter: Kommen wir damit mehr oder weniger zum Schluss? Alfred, hättest du besondere Anliegen an die Rolle der ÖSG heute?

Alfred Franz: Ja, die ÖSG heute ist für mich eine eindrucksvolle Sache. Ich bin damit wirklich glücklich und bin froh, dass sich alles so entwickelt hat. Sie ist wirklich eine vitale wissenschaftliche Gesellschaft geworden, sowohl was die eigenen Veranstaltungen betrifft als auch die Teilnahme an internationalen Veranstaltungen. In Deutschland ist es ein gängiges Urteil: Die ÖSG, die gibt es, die machen was. Ist das kein gutes Zeichen?

Zwei kleine Desiderata hätte ich vielleicht noch: Eines ist etwas mehr Naives, ich beziehe natürlich als nicht Mitgliedsbeitrag zahlendes Ehrenmitglied weiterhin die Zeitschrift der Statistischen Gesellschaft, und zwar in Papierform. Ich hoffe, dass sie nicht nur wegen mir alleine noch gedruckt wird. Und so blättere ich sie durch, und am Schluss bin ich immer leicht deprimiert, weil ich finde keinen einzigen Artikel, den ich wirklich verstehe. Ich weiß nicht, ob man das in dieses Interview hineinschreiben darf, und bin damit wahrscheinlich sowieso disqualifiziert. Aber es ist ein ganz anderes, um nicht zu sagen fremdes Milieu, das da heute statistisch behandelt wird. Das ist das Eine. Und das Zweite ist eine historische Reminiszenz. In meiner statistischen Jugend gab es dann und wann große statistische Vorträge. Ich erinnere mich noch an einen von Prof. Tintner, ein aus Österreich vertriebener großer Ökonometriker, eine große statistische Kapazität. Und so an einige andere Vorträge, die hat man am Statistischen Institut an der Uni Wien gehalten, in einem regelrechten Hörsaal. Das war schon auch eine feine Sache, dass es von den Arbeitskreisen abgehoben solche großen Vorträge gab. Wäre schön, wenn man das dann und wann wieder machen könnte. Ich würde meinen, Geld genug hat die Gesellschaft, die könnte sich das leisten. Ich habe keinen konkreten Vorschlag, wen man da einladen soll, außer mir selber - bitte das nicht zum Protokoll - das wären meine Ideen.

Josef Richter: Vielleicht so quasi zum Abschluss. Wie seinerzeit bei der so verdienten Verleihung. der Ehrenmitgliedschaft erwähnt: Es zeichnet Alfred Franz aus, dass er sich mit den wirklich fundamentalen Fragen der beschreibenden Amtlichen Statistik intensiv auseinandersetzt. Gibt es da noch ein Thema, abgesehen vom Staat, dessen Behandlung in der Statistik ja ein nachgerade lebensbegleitendes Thema ist, das dich derzeit intensiv beschäftigt? 
Alfred Franz: Diese Formulierung liebe ich, die trifft alles, was mich in meiner Vergangenheit so „umgetrieben“ hat. Ein etwas abseits davon liegendes Thema, aber nicht ganz anderer Art, könnte ich schon ins Treffen führen. Womit ich mich derzeit beschäftige, das ist der Begriff der „Statistischen Zahl“. Interessanterweise finde ich dazu überhaupt keine Referenzen, was allein schon bedeutet: Oha, entweder ist es ein Nullthema, oder ist es ein Topthema, eines von beiden. Mich fasziniert das jetzt einmal, und ich schreib daran, natürlich nicht mit dem Nachdruck der jüngeren Leute, und ich bin daher noch nicht ganz fertig damit. Sobald der Text im Feinschliff vorliegt, werde ich nicht zögern, ihn zuerst einmal meinen Interviewchefs zur Verfügung zu stellen. Könnte mir aber vorstellen, dass wir dazu in einem geneigten, kleineren Kreis einmal eine Diskussion hätten: Was bedeutet das eigentlich? Was lässt sich dazu überhaupt sagen? Mir scheint es also keineswegs so klar, was das ist, eine "Statistische Zahl“. Das fängt schon damit an, dass wir in der Mathematik zehnerlei Zahlenarten haben, aber keine „Statistische Zahl“, sie kommt dort einfach nicht vor. Natürlich, kann ja gar nicht vorkommen, weil sie ist ja keine von diesen zehn Zahlen. Aber immerhin, es ist eine Zahl, die ganz bestimmte Qualitäten hat, und da kann man meditieren, wo überall so was vorkommt, und was es jeweils für eine Bedeutung hat. Aber ich will das jetzt nicht in extenso ansprechen. Es beschäftigt mich und vielleicht ist es das letzte, was ich in meinem statistischen Leben noch anstelle, und würde mich groß freuen, wenn ich das irgendwie noch unters Volk bringen kann.

Josef Richter:Ich glaube, das ist ein nahezu idealer Schluss für unser Interview. Vielen Dank für das nette, stimulierende Gespräch.

Alfred Franz: Auch ich habe zu danken, nicht zuletzt für die Geduld, die ihr mit mir gehabt habt.

Die Interviewer bedanken sich herzlich bei Gabriele Mack-Niederleitner und Sandra ZeimlYudytskiy für die Transkription.

\section{Literatur}

Die folgende Aufstellung umfasst die wichtigsten, im Interview angesprochenen Arbeiten.

Franz A. (1984). „Von den ontologischen Konzepten in der amtlichen Statistik“. Mitteilungsblatt der Österreichischen Statistischen Gesellschaft 54/1984. I2I - I38

Franz A. (1985). "Estimates of the Hidden Economy in Austria on the Basis of Official Statistics". The Review of Income and Wealth 4/1985. 325 - 336

Franz A. (Hrsg. I986): „Statistische Ausbildung heute, Symposion der Österreichischen Statistischen Gesellschaft, Linz, 2. - 5. Oktober 1986“. Schriftenreihe der ÖSG, Orac, Wien 1988

Franz A. (1993a). "Some thoughts on systems of regional accounts". Statistical Journal of the United Nations ECE I/1993. 17 - 45

Franz A. (1993b). „Entwicklung einer Öko-VGR in Österreich: Input-Output als Alpha und Omega“. In: Schnabl H. (Hrsg.): Ökointegrative Gesamtrechnung. Walter de Gruyter, Berlin, New York 1993. 7I $-9^{3}$ 
Franz A. (1994). „Volkswirtschaftliche Gesamtrechnungen. Das statistische System der Makroökonomie“. In: Österreichisches Statistisches Zentralamt (Hrsg.): Österreichische Studien zur amtlichen Statistik, Nr. I. Wien 1994

Franz A. (1995). „Das weibliche Bruttoinlandsprodukt in der volkswirtschaftlichen Gesamtrechnung“. In: Der volkswirtschaftliche Wert der Hausarbeit; Bewertungsmodelle und politische Konsequenzen; Bundesministerium für Umwelt, Jugend und Familie; Wien 1995. 7- I3

Franz A. (1996a). „Regionale Gesamtrechnungen: Dürfte man, was man tut? Tut man, was man dürfte?“. In: Reich U. P., Stahmer C., fVoy C. (Hrsg.): Kategorien der Volkswirtschaftlichen Gesamtrechnungen, Metropolis, Marburg 1996. 205 - 235

Franz A. (1997). „A Class Society of National Accounts Compilers? Considerations on Certain Requirements and Some Consequences of the Transition to the New System". Österreichische Zeitschrift für Statistik I/1997. 53 - 58

Franz A., Rainer N. (Eds. 1986). "Problems of Compilation of Input-Output Tables, Proceeding of the International Meeting organized by the Austrian Statistical Society, Baden near Vienna, Austria, I925 May, I985“. Schriftenreihe der ÖSG, Orac, Wien 1986

Franz A., Rainer N. (Eds. 1989). "Compilation of Input-Output Data, Proceedings of the 2nd International Meeting on problems of Compilation of Input-Output Tables, organized by the Austrian Statistical Society, Baden near Vienna, Austria, ı3-ı9 March, ı988”. Schriftenreihe der ÖSG, Orac, Wien 1989

Franz A., Richter J. (2009). „Eine kurze Geschichte der Volkswirtschaftlichen Gesamtrechnungen in Österreich“. In: Voy C. (Hrsg.): Kategorien der Volkswirtschaftlichen Gesamtrechnungen - Zur Geschichte der Volkswirtschaftlichen Gesamtrechnungen nach 1945. Metropolis, Marburg 2009• 475 502

Franz A., Stahmer C. (Hrsg. 1993). "Approaches to Environmental Accounting, Proceedings of the IARIW Conference on Environmental Accounting, Baden I991"; Physica, Heidelberg $199^{3}$

Rainer N. (Hrsg. 1999) „Österreichs Statistik in der Europäischen Integration“. In: Österreichisches Statistisches Zentralamt (Hrsg.): Österreichische Studien zur amtlichen Statistik, Nr. 2. Wien 1999

Richter J. (2002). "Kategorien und Grenzen der empirischen Verankerung der Wirtschaftsforschung". Lucius, Stuttgart 2002

Stiglitz J., Sen A., Fitoussi J. (2009). "Report by the Commission on the Measurement of Economic Performance and Social Progress". https://ec.europa.eu/eurostat/documents/II8025/II 8 I23/Fitoussi+Commission+report

United Nations Statistical Office $(1975)$. „Towards a System of Social and Demographic Statistics (SSDS)". ST/ESA/SER.F/18, New York, United Nations 1975 


\section{Affiliation:}

Alfred Franz

Vormals Österreichisches Statistisches Zentralamt, ab 2000 Statistik Austria

E-Mail: alfred.franz@chello.at

URL: vgr.cc/cms/index.php

Norbert Rainer

Vormals Österreichisches Statistisches Zentralamt, ab 2000 Statistik Austria

E-Mail: NorbertRainer@gmx.at

Josef Richter

Vormals Bundeskammer der gewerblichen Wirtschaft, Wirtschaftskammer Österreich E-mail: josef.richter@aon.at 
Ein Interview mit Alfred Franz 